\title{
Avaliação do risco de queda e da demanda atencional em idosos submetidos a um protocolo cinesioterapêutico de dupla tarefa
}

\author{
Assessment of risk of falling and attentional demand in elderly submitted to a protocol \\ kinesiotherapeutic dual task
}

\begin{abstract}
Carlos Drummond Alcântara da Silva1, Sarah Tarcisia Rebelo Ferreira de Carvalho', Maria Claudia Gonçalves ${ }^{1}$, Cândida Helena Alves, Karla Virginia, Bezerra de Castro Soares ${ }^{1}$
\end{abstract}

\begin{abstract}
Resumo: Introdução: Grande parte das quedas em idosos ocorre ao andar e realizar ações secundárias, pois a dupla tarefa aumenta a demanda atencional e motora necessárias para controle e manutenção do equilíbrio. Objetivo: avaliar os efeitos de um protocolo cinesioterapêutico de dupla tarefa sob o risco de quedas relacionada à mobilidade e o equilíbrio corporal dinâmico e à demanda atencional em idosos. Materiais e métodos: Estudo de intervenção com 40 idosas ingressantes de um programa assistencial ao idoso, submetidos a um protocolo cinesioterapêutico de dupla tarefa envolvendo simultaneamente tarefas motoras e cognitivas por 16 semanas, com duas sessões semanais de 50 minutos. Na coleta de dados, empregou-se o teste Timed Up and Go (TUG Simples) para avaliar o risco de queda e o Timed Up and Go cognitivo (TUG Cognitivo) para avaliar a demanda atencional relacionada ao risco de quedas. Para a análise estatística, utilizou-se o programa SPSS 18.0. Na estatística descritiva, as variáveis foram apresentadas em média, frequência relativa e percentual. Para a análise do efeito do protocolo sobre a capacidade atencional e risco de cair, utilizou-se o teste de Wilcoxon, sendo considerado significativo $p<0$, 05.Resultados: Quanto ao risco de queda, o tempo médio de execução antes da intervenção foi 23,09 segundos e depois do protocolo,19,73 segundos $(p<0,0001) .40 \quad(100 \%)$ dos idosos estudados, apresentavam risco moderado de queda antes da intervenção e após o protocolo, 36 idosos (90\%), apresentaram baixo risco de queda. Quanto à capacidade atencional relacionada ao risco de queda, o tempo médio de execução dos testes antes da intervenção foi de 26,87 segundos e depois da intervenção foi de 19,80 segundos $(p<0,0001)$. Antes da aplicação do protocolo, 39 idosos $(97,50 \%)$ apresentavam alto risco de quedas e após a intervenção, 34 idosos (85\%) apresentaram baixo risco de queda. Conclusão: 0 protocolo de dupla tarefa utilizado diminuiu o risco de queda nos idosos estudados, tanto relacionado ao aspecto da mobilidade funcional isolada, quanto associada ao aspecto cognitivo. Sugere-se, assim, o a adição de exercícios de dupla tarefa na prática clínica e nas práticas de atividades físicas.
\end{abstract}

\section{Palavras-chave: Quedas,Dupla Tarefa,Idosos.}

Abstract: Introduction: Most of falls in the elderly occurs when walking and perform secondary actions, as the dual task increases attentional demand and motor needed to control and maintain balance. Objective: To evaluate the effects of a kinesiotherapeutic protocol dual task under the risk off alls related to mobility and dynamic body balance and attentional demand in the elderly. Materials and methods: intervention study with a sample of 40 fresh men elderly of an assistance program for the elderly, subject to a kinesiotherapeutic protocol dual task involving both motor and cognitive tasks for 16 weeks, with two weekly sessions of 50 minutes. Data collection, we used the Timed Up and Go test (TUG Simple) to assess the risk of falling and the Timed Up and Go cognitive (TUG Cognitive) to evaluate the attentional demand related to fall risk. A descriptive analysis of the quantitative variables were expressed as mean and standard deviation and qualitative variables presented by absolute and percentage frequencies. Data were analyzed using Stata 12.0. Results: The risk of falling, the average execution time before intervention was 23.09 seconds, then the protocol, 19.73 seconds with $\mathrm{p}<0.0001 .40(100 \%)$ of the elderly, had moderate risk of falling before the intervention and after the protocol, 36 elderly $(90 \%)$ have a low risk of falling. As for attentional capacity related to the risk of falling, the average execution time of testing before intervention was 26.87 seconds, and after the intervention was 19,80 seconds. Before the application of the Protocol, 39 elderly $(97.50 \%)$ were at high risk of falls and after the intervention, 34 subjects $(85 \%)$ showed a low risk of falling. Conclusion: the dual task of protocol used decreased the risk of falling in the elderly studied, both related to the aspect of the isolated functional mobility, as associated with the cognitive aspect. It is suggested, therefore, the addition of dual task in clinical practice exercise and physical activity practice

Keywords: Risk of falling. Double Task. Elderly.

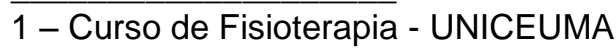




\section{Introdução}

O envelhecimento populacional se configura como a maior conquista da sociedade moderna, pois a expectativa de vida nos últimos 100 anos foi maior que em qualquer momento da história. $\mathrm{O}$ número de idosos com 60 anos ou mais passou de 15,5 milhões para 23,5 milhões de pessoas e o Índice de Envelhecimento em 2012 atingiu 51,8 no Brasil, comparado a 31,7 em 2001, superior à média mundial que é de 48,2.Segundo o último censo, a população de idosos no Maranhão alcançou o número de 74.765 de idosos, mais que o dobro da população de crianças e adolescentes ${ }^{1}$.

No entanto, um cenário dispare acompanha 0 fenômeno do envelhecimento. Se por um lado o corpo passou a ser medida de identidade pessoal e até da personalidade com a longevidade sintetizando a saúde, bemestar e a autonomia funcional sendo sinônimo de qualidade de vida, por outro, os Indicadores Sócio-demográficos e de Saúde no Brasil mostram que as doenças crônicas chegam a atingir $75,5 \%$ dos idosos do País².

Destaca-se que as alterações sistêmicas estão atreladas ao envelhecimento, a exemplo de disfunções do sistema musculoesquelético, nervoso, somatossensorial, visual, vestibular e cardiorrespiratório, além de déficits cognitivos, que podem ainda ser agravados pela presença de doenças crônicas e uso exacerbado de medicamentos que podem acarretar redução da funcionalidade e perda da autonomia funcional, aumentando assim o risco de quedas ${ }^{3}$.

Em se tratando de quedas, os dados são alarmantes e clamam por medidas efetivas. Dados mostram que todos os anos mais de um terço das pessoas com 65 anos ou mais caem no mundo, com possibilidade de recorrência de quedas em metade dos casos. No Brasil, o número de quedas em idosos é de no mínimo 350 milhões por ano, sendo que $50 \%$ resultariam em algum tipo de lesão 4 .

A queda geralmente está associada ao declínio natural de desempenho do sistema de controle postural e diminuição das capacidades cognitivas, a exemplo da capacidade atencional, resultando na diminuição do tempo de reação e proteção a queda.Isto porque a atenção dividida na realização concomitante de várias atividades exige maior demanda atencional acarretando maior dificuldade no desempenho motor e no controle do equilíbrio aumentando assim a probabilidade de cair ${ }^{5}$.

Levantar, caminhar e sentar são atividades rotineiras e cotidianas comumente realizadas em associação a outras tarefas motoras, visuais e/ou cognitivas, o que faz da capacidade de realizar múltiplas tarefas algo extremamente vantajoso e, na maioria das vezes, um pré-requisito para uma vida normal ${ }^{6}$.

Em se tratando do idoso, este é capaz de manter o equilíbrio corporal quando está concentrado em uma atividade específica, mas pode não apresentar o mesmo padrão de execução quando precisa realizar múltiplas tarefas simultaneamente. Isto ocorre porque, com o aumento da idade cronológica, o corpo humano passa por um período de transformações que geram declínio de algumas capacidades físicas, tais como a diminuição da flexibilidade, agilidade, coordenação, mobilidade articular e, principalmente, o equilíbrio ${ }^{7}$.

Considera-se assim a queda um evento complexo com causas multifatoriais, que tem relação direta com a falta de equilíbrio e diminuição do desempenho físico e todas as possíveis causas devem ser tratadas como medida cautelar.Com base nesta premissa, o objetivo deste estudo foi avaliar os efeitos de um protocolo cinesioterapêutico de dupla tarefa sob o risco de quedas relacionada à mobilidade e o equilíbrio corporal dinâmico à demanda atencional em idosos. 


\section{Material e Método}

Este é um estudo de intervenção desenvolvido respeitando ás Norma estabelecidas na Resolução № 466, de 12 de dezembro de 2012 do Conselho Nacional de Saúde com relação à Realização de Pesquisa em Seres Humanos, tendo seu projeto já aprovado pelo Comitê de Ética e Pesquisa (CEP), da Universidade CEUMA pelo parecer de número 541.619.

O estudo foi realizado no Núcleo de Pesquisa em Reabilitação FuncionalNUPERF, na Universidade Ceuma em São Luís, MA. Para compor a amostra, sorteou-se 40 idosos iniciantes no programa Uniceuma sem fronteiras, projeto de extensão multiprofissional que atende idosos da comunidade desde o ano de 2010.

Como critério de inclusão, considerou-se: indivíduos com idade entre 60 e 65 anos, do gênero feminino, sedentário à pelo menos seis meses; não pertencente a nenhum outro grupo de pratica de atividades físicas, de lazer ou intelectuais; apresentando-se lúcido e hígido, de acordo com encaminhamento médico. Como critério de exclusão considerou-se idosos com diagnóstico de doenças neurológicas, distúrbios do aparelho vestibular, deficiência visual não corrigida, paralisias de qualquer etiologia e alterações ortopédicas, que impossibilitasse se manter em ortostatismo e se locomover sem assistência, e o uso de fármacos que comprometam a fidedignidade dos testes.

Depois de considerados os critérios, a amostra foi submetida a um pré-teste, utilizando para a coleta de dados os seguintes instrumentos:

a) Timed Up and Go (TUG Simples) teste que avalia a mobilidade e o equilíbrio corporal dinâmico, além do risco de quedas em idosos, validado para o Brasil por Cabral ${ }^{8}$. Este teste quantifica, através de um cronômetro, em quantos segundos ele levanta de uma cadeira padronizada com apoio e braços e de aproximadamente $46 \mathrm{~cm}$ de altura e braços de $65 \mathrm{~cm}$ de altura, caminha 3 metros, vira, volta rumo à cadeira e senta novamente.

A velocidade que 0 paciente desempenha no teste está relacionada ao risco de queda, sendo que um tempo menor de 20 segundos indica um pequeno risco de cair. Um escore entre 20 e 30 segundos indica risco moderado e um escore de mais de 30 segundos indica um alto risco de queda.

b) Timed Up and Go Cognitivo (TUG Cognitivo): trata-se da versão modificada do TUG, associada a uma tarefa cognitiva para avaliar a influência da demanda atencional no equilíbrio corporal de idosos ${ }^{10}$. O procedimento de aplicação é o mesmo do TUG, acrescentando-se a evocação de nomes de animais precedido do teste de Fluência Verbal.

c) Teste de fluência verbal Categoria animal foi realizado junto aos testes TUG e TUG Cognitivo. Este teste avalia a memória semântica no que se refere ao conhecimento geral prédefinido. Para esta avaliação, o paciente foi solicitado a enumerar o máximo de animais em um minuto cronometrado, enquanto 0 pesquisador anotava 0 número de animais dito pelos pacientes. Foi obedecido o critério da categoria semântica de contar um ponto para cada nome de animal para masculino, ou seja, boi e vaca marcava-se somente um ponto ${ }^{11,12}$.

Em seguido, a amostra foi submetida a um protocolo cinesioterapêutico de dupla tarefa. O protocolo de exercícios utilizado teve como base o protocolo original criado por Soares $^{13}$, com duração de dezesseis semanas, em uma frequência de duas vezes por semana, durante 50 minutos por sessão. Ao protocolo original, foram inseridas ao longo das sessões demandas atencionais diversas associadas ao exercício como estímulos visuais, auditivos e de raciocínio lógico e memória. O protocolo de exercícios 
distribui-se em quatro níveis distintos, a saber:

a) Nível I: (1 $1^{a}$ semana) Exercícios de consciência corporal, associada à compreensão dos padrões respiratórios, associados a demandas envolvendo comando verbal e tempo de resposta. Exemplo: Evocação do nome de animais, cores, e nomes próprio durante os exercícios.

b) Nível II: (2 $2^{\underline{a}}$ a $4^{\underline{a}}$ semana) Exercícios leves, com movimentos de mínima amplitude com sutil contração isométrica local, associados a demandas envolvendo comando verbal e tempo de resposta. Exemplo: Atividades de atenção como contar de um a cem ao contrário, durante a execução dos exercícios.

c) Nível III: (5 $5^{\underline{a}}$ a $10^{\underline{a}}$ semana) Exercícios leves, envolvendo movimentos de pequena amplitude, contração isométrica global associada à contração isotônica livre (sem carga), associados a demandas envolvendo atenção visoespacial. Exemplo: Seguir traçados progressivamente mais complexos no chão durante a execução dos exercícios.

d) Nível IV: (11aa a $16^{\underline{a}}$ semana) Movimentos de grande amplitude com contração isométrica global máxima e isotônica com carga, com evolução no nível de complexidade associados a demandas envolvendo atenção viso- espacial e comando verbal. Exemplo: uso de imagens de animais, situações rotineiras e de fatos históricos foram mostradas em uma tela para que fossem identificadas durante a realização dos exercícios.

Após 16 semanas de intervenção, foi realizado um pós-teste para obter os dados finais relativos à mesma, utilizando-se os mesmos instrumentos empregados no pré-teste.

Para a análise estatística, utilizou-se o programa SPSS 18.0. Na estatística descritiva, as variáveis foram apresentadas em média, frequência relativa e percentual. Para a análise do efeito do protocolo sobre a capacidade atencional e risco de cair, utilizou-se o teste de Wilcoxon, sendo considerado significativo $p<0,05$.

\section{Resultados}

Ao comparar o desempenho dos participantes antes da intervenção proposta em relação aos testes TUG Simples e ao TUG Cognitivo, percebe-se que 40 idosos (100\%) apresentavam médio risco de queda para o TUG Simples e $39(97,50 \%)$ tinham alto risco de queda com base no TUG Cognitivo (Tabela 1).

Tabela 1 - Distribuição dos idosos investigados quanto ao risco de queda com base nos testes TUG Simples ${ }^{1}$ e TUG Cognitivo² antes da intervenção, São Luís, Maranhão, 2014.

\begin{tabular}{lcccc}
\hline Risco de Queda & \multicolumn{2}{c}{ TUG Simples $^{1}$} & \multicolumn{2}{c}{ TUG Cognitivo² } \\
& $\mathrm{n}$ & $\%$ & $\mathrm{n}$ & $\%$ \\
\hline Baixo Risco & - & - & - & - \\
Médio Risco & 40 & 100,0 & 01 & 02,50 \\
Alto Risco & - & - & 39 & 97,50 \\
\hline TOTAL & 40 & 100 & 40 & 100 \\
\hline 1Timed Up and Go Test & & & \\
2Time Up and Go Cognitivo & & &
\end{tabular}


Tabela 2 - Distribuição dos idosos investigados quanto ao risco de queda antes e após a intervenção com protocolo de dupla tarefa, São Luís, Maranhão, 2014.

\begin{tabular}{lcccc}
\hline $\begin{array}{l}\text { Risco de Queda } \\
\text { (TUG') }\end{array}$ & \multicolumn{2}{c}{$\begin{array}{c}\text { Antes da } \\
\text { Intervenção }\end{array}$} & \multicolumn{2}{c}{$\begin{array}{c}\text { Depois da } \\
\text { Intervenção }\end{array}$} \\
& $\mathrm{n}$ & $\%$ & $\mathrm{n}$ & $\%$ \\
\hline Baixo Risco & - & - & 36 & 90,00 \\
Médio Risco & 40 & 100,0 & 04 & 10,00 \\
\hline TOTAL & 40 & 100 & 40 & 100 \\
\hline \multicolumn{1}{r}{${ }^{1}$ Timed Up and Go Test } & & &
\end{tabular}

Em relação ao risco de queda avaliado pelo TUG Simples, o tempo médio de execução antes da intervenção foi 23,09 segundos e depois do protocolo, 19,73 segundos, com $p<0,0001$. Os resultados da tabela 2 demonstram que todos os 40 idosos estudados $(100 \%)$, apresentavam risco moderado de queda antes da intervenção. Após o protocolo de dupla tarefa, apenas 04 idosos (10\%) mantiveram-se com risco moderado de queda, enquanto 36 idosos $(90 \%)$, apresentaram baixo risco de queda.

Quanto à Capacidade Atencional relacionada ao risco de queda (TUG Cognitivo), o tempo médio de execução dos testes antes da intervenção foi de 26,87 segundos e depois da intervenção foi de 19,80 ( $p<0,0001)$.

Constatou-se também que antes da aplicação do protocolo de dupla tarefa, a maioria dos idosos estudados $(n=39$; $97,50 \%$ apresentavam alto risco de quedas segundo a classificação referente ao TUG cognitivo. Após a intervenção, 34 idosos $(85 \%)$ apresentaram baixo risco de queda. Com aplicação do teste de contingência para variáveis independentes ficou definido $p<0,0001$ (Tabela 3).

\section{Discussão}

Neste trabalho, o risco de queda nos idosos estudados foi analisado através de dois testes. O primeiro, Timed Up and Go (TUG Simples), avalia este risco mediante a avaliação da mobilidade e do equilíbrio corporal dinâmico. O segundo, consiste no TUG associado à tarefa cognitiva, relacionada à demanda atencional.

Tabela 3 - Distribuição dos idosos investigados quanto à capacidade atencional relacionada ao risco de queda antes e após a intervenção com protocolo de dupla tarefa, São Luís, Maranhão, 2014.

\begin{tabular}{|c|c|c|c|c|}
\hline \multirow{2}{*}{$\begin{array}{l}\text { Capacidade Atencional } \\
\text { relacionada ao risco de } \\
\text { queda } \\
\left.\text { (TUG Cognitivo }{ }^{1}\right)\end{array}$} & \multicolumn{2}{|c|}{$\begin{array}{l}\text { Antes da } \\
\text { Intervenção }\end{array}$} & \multicolumn{2}{|c|}{$\begin{array}{l}\text { Depois da } \\
\text { Intervenção }\end{array}$} \\
\hline & $\mathrm{n}$ & $\%$ & $n$ & $\%$ \\
\hline Baixo Risco & 01 & 2,50 & 34 & 85,00 \\
\hline Médio Risco & 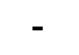 & - & - & - \\
\hline Alto Risco & 39 & 97,50 & 06 & 15,00 \\
\hline TOTAL & 40 & 100,00 & 40 & 100,00 \\
\hline
\end{tabular}

1 Time Up Go Cognitivo 
Verificou-se inicialmente que antes da intervenção proposta, todos os idosos apresentavam médio risco de queda para o TUG Simples enquanto $97,50 \%$ deles tinham alto risco de queda considerando o TUG Cognitivo. Isto indica que a adição da tarefa cognitiva diminuiu 0 desempenho dos participantes e demandou a necessidade de um tempo maior para completar a tarefa proposta pelo TUG, sugerindo, assim, o impacto negativo da tarefa cognitiva para o risco de queda dos idosos estudados.

Esse resultado é semelhante ao achados do estudo de Melzer e Oddsson $^{14}$ sobre 0 efeito da tarefa cognitiva na execução do passo voluntário em idosos e jovens saudáveis, que indicaram a tarefa cognitiva como um importante fator contribuinte para perda de equilíbrio e ocorrência de quedas nesta população. De forma similar, Carmelo e Garcia ${ }^{15}$ realizaram um estudo com o objetivo de avaliar e comparar o equilíbrio dinâmico sob a condição de tarefa única e de dupla-tarefa de idosas praticantes de exercício físico regular e idosas sedentárias, e concluíram que em ambos os grupos, o tempo para realização do TUG Cognitivo foi significativamente maior em relação ao TUG Simples.

A pesquisa de Hatzitaki e Amiridis $^{16}$ também demonstrou que a diminuição da capacidade atencional é evidentemente acentuada em idosos, e que essa diminuição tem significante responsabilidade no aumento do risco de quedas desses idosos, dados que corroboram com os resultados encontrados na presente pesquisa.

Considera-se que a diminuição da capacidade de atenção acarreta a dificuldade de controle de equilíbrio mediante um aumento na demanda atencional que pode estar relacionada ao encolhimento da área pré-frontal do cérebro, visto que esta área está fortemente relacionada com funções de execução, tais como a gestão de múltiplas tarefas, o que acaba por aumentar o risco de quedas associado ao envelhecimento ${ }^{17}$.

Estudos realizados nos Estados Unidos e na Europa mostram que aproximadamente $1 / 3$ da população acima de 65 anos sofreu pelo menos uma queda durante o ano de 2012. Entre os idosos que sofreram as quedas, de $10-15 \%$ relatam que estavam realizando duplas tarefas ${ }^{18}$.

Neste sentido destaca-se a importância de se avaliar e buscar intervenções que considere o aspecto da tarefa cognitiva para que se possa realizar ações de prevenção de quedas efetivas. Principalmente, visto que em geral, as atividades de vida diária são compostas pela realização simultânea de uma tarefa motora (andar, levantar, sentar) e de uma tarefa cognitiva.

Em relação aos efeitos do protocolo de dupla tarefa sob o risco de quedas nos idosos avaliados pelo TUG Simples e pelo TUG Cognitivo, percebeu-se diminuição significativa $(p<0,0001)$ em relação ao tempo médio de execução de ambos os testes antes e após o protocolo.

Ao realizar uma análise comparativa entre os dois testes antes e depois da intervenção, constata-se que antes da aplicação do protocolo, o tempo médio de execução do TUG Simples e do TUG Cognitivo eram, respectivamente, 23,09 segundos e 26,87 segundos. Após a intervenção, este tempo passou a ser, respectivamente, 19,73 segundos e 19,80 segundos. Desta forma percebe-se que após a aplicação do protocolo de dupla tarefa, a diferença de tempo necessário para realizar 0 teste com e sem o adicional da tarefa atencional diminuiu, de forma a sugerir que a demanda atencional passou a 
ter menor influência na mobilidade e equilíbrio funcional dos idosos estudados, e por consequência, menor interferência no risco de queda dos mesmos.

Percebeu-se ainda que dos 40 idosos (100\%) que apresentavam risco moderado de queda conforme o TUG Simples antes da intervenção, 36 $(90 \%)$ diminuíram este risco sendo reclassificados com baixo risco após a intervenção. Em relação ao TUG Cognitivo, percebeu-se que dos 40 idosos (100\%) antes classificados com risco moderado ou alto de queda, 34 $(85 \%)$ apresentaram baixo risco de queda após a intervenção.

Neste contexto, verificou-se no presente estudo um significativo aumento na capacidade motora, como também atencional dos idosos submetidos ao protocolo, assim como uma consequente diminuição no risco de cair, comprovado através da diminuição de tempo de execução das tarefas estabelecidas.

Destaca-se que a integridade dos mecanismos posturais para lidar com a instabilidade durante a realização de duplas tarefas sofre deterioração com o passar dos anos e somadas as mudanças estruturais no sistema locomotor, como a diminuição da força e da flexibilidade, que acabam por influenciar negativamente padrões cinemáticos e cinéticos da marcha ${ }^{19}$.

Sobre estes aspectos, salientase que a atividade física constitui em excelente instrumento de saúde em qualquer faixa etária, mas em especial no idoso, induzindo várias adaptações fisiológicas e psicológicas, sendo instrumento essencial para diminuição do risco de quedas ${ }^{20}$. Park ${ }^{21}$ também defende que os exercícios em geral reduzem o risco de quedas, uma vez que aumentam a força muscular e melhoram o equilíbrio e marcha, mas salienta que os exercícios são mais eficazes quando são associados a protocolos de dupla tarefa, já que contribuem significativamente para um aumento na capacidade atencional.

Desta forma, a prática regular de exercícios físicos associados a programas de dupla tarefa para a população idosa é considerada um importante fator de intervenção e prevenção de quedas, já que além de aumentar força muscular e melhorar o controle postural, estimulam aumento da capacidade atencional ${ }^{19}$. Por consequência, treinamentos específicos utilizando estimulação cognitiva e funcional, causam diminuição significativa do risco de quedas em idosos, sendo demonstrado diferença notável entre idosos submetidos ao protocolo de treinamento $e$ idosos que não passaram pelo programa quanto ao equilíbrio, atenção e força muscular ${ }^{22}$.

Destaca-se que com o treinamento adequado, é possível diminuir a interferência negativa entre as tarefas fazendo com que o desempenho na tarefa-dupla melhore abordando uma possível interação destes dois componentes ${ }^{23}$.

Assim, o desenvolvimento de programas específicos de reabilitação associados a protocolos de dupla tarefa com o objetivo de manter ou melhorar o desempenho físico e cognitivo no processo de envelhecimento vem sendo efetivos não somente para evitar quedas, mas também para proporcionar, no caso de ocorrência destas, a capacidade funcional necessária para erguer-se, reparar os danos e em longo prazo, readquirir confiança e independência ${ }^{24}$.

\section{Conclusão}

Os resultados do estudo demonstraram que 0 protocolo de dupla tarefa utilizado diminuiu o risco de queda nos idosos estudados, tanto 
relacionado ao aspecto da mobilidade e equilíbrio dinâmico isolado, quanto associado ao aspecto cognitivo.

Desta forma, sugere-se o incentivo da adição de exercícios de dupla tarefa na prática clínica e nas práticas de atividades físicas regulares como uma forma de melhorar o desempenho funcional e prevenir possíveis quedas em idosos.

\section{Referências}

1. IBGE. Instituto Brasileiro de Geografia e Estatísticas. Projeção da População do Brasil e das Unidades da Federação, por Sexo e Idade para o Período 2000-2030, 2013.

2. IBGE. Instituto Brasileiro de Geografia e Estatísticas, Indicadores Sociodemográficos e de Saúde no Brasil, 2009.

3. Barbosa JMM et al. feito da realização simultânea de tarefas cognitivas e motoras no desempenho funcional de idosos da comunidade. Fisiote 2015. 8 (4): 374-379.

4. Dantas EL, Brito GEG, Lobato, IAF. Prevalência de quedas em idosos adscritos à estratégia de saúde da família do município de João Pessoa. Rev APS. 2012 jan/mar; 15(1): 67-75.

5. Gonçalves D et al. Comparação do tempo de reação simples de idosos e adolescente de Cruz Alta, Biomotriz. 2013. 7(1): 52-62.

6. Sulwan R. Effect of practice and span length on the dual- task coordination executive test. Braz J Med Biol Res. 2009; 32(10): 1263-8

7. Mann L et al. Investigação do equilíbrio corporal em idosos. Rev Bras Geriatr Gerontol. 2008; 11(2):1809-23.
8. Cabral ALL. Tradução e validação do teste Timed Up and Go e sua correlação com diferentes alturas da cadeira. 102f.2011. Dissertação (Mestrado em Gerontologia) Programa de Pós Graduação em Gerontologia, Universidade Católica de Brasília, Brasília, DF, 2011.

9. Kato-Narita EM, Nitrini $R$, Radanovic M. Assessmentof balance in mild and moderate stages of Alzheimer's Disease: implications on fall sand functional capacity. Arq Neuropsiquiatr. 2011;69(2A):202-7.

10. Caixeta Giovanna Cristina dos Santos, Doná Flávia, Gazzola Juliana Maria. Processamento cognitivo e equilíbrio corporal em idosos com disfunção vestibular. Braz. j. otorhinolaryngol. 2012. 78(2):87-95

11. Rodrigues AB, Yamashita ET, Chiappetta ALML. Teste de fluência verbal no adulto e no idoso: verificação da aprendizagem verbal. Rev. CEFAC. 2008 .10(4): 443-451.

12. Xavier FMF et al. Episódio depressivo maior, prevalência e impacto sobre qualidade de vida, sono e cognição em octogenários. Rev. Bras. Psiquiatr. 2001. June; 23(2):62-70.

13. Soares MA, Sacchelli T. Efeitos da cinesioterapia no equilíbrio de idosos. Rev Neurocienc. 2008;16(2):97-100.

14. Melzer I, Oddsson LI. The effectof a cognitive task on voluntary step execution in healthy elderly and young individuals. J Am Geriatr Soc. 2004;52(8):1255-62.

15. Carmelo VVB, Garcia PA. Avaliação do equilíbrio postural sob condição de tarefa única e tarefa dupla em idosas sedentárias e não 
sedentárias. Acta Fisiátr. 2011;18(3):136-140.

16. Hatzitaki, V.; Amiridis, I.G. Aging effect son postural responses to self-imposed balance pertubations. Gait and Posture. 2009. 22(3): 25057.

17. Shimada, H. Obuchi, S. Relationship with the dynamic balance function during standing and walking. Physical medicine. 2010; 82:511-516.

18. Ryynanen P. Incidence and Risk Factors for Falling Injuries among the Elderly. Acta Universitatis Ouluensis, Series D.2013; 265.

19. Bassey EJ. Physical capabilities, exercise and aging. Reviews in Clinical Gerontology. 2010.7:289297.

20. Skelton, D.A. Effects of physical activity on postural stability. Age andageing. 2011. 30(4), 33-39.

21. Park H, Kim KJ. Effect of combined exercise training on bone, body balance, and gaitability: a randomized controlled study in community- dwelling elderly women. J. Bone miner. Metab. 2008; 26 (3): 254.9.

22. Maciel AC, Guerra RO.

Prevalência e fatores associados ao déficit de equilíbrio em idosos.

Revista Brasileira de Saúde e Ciência.2009;13(1):37-44.

23. Bueno MEB, Andrello ACR, Terra MB. Efetividade da Fisioterapia com Treinamento de Dupla Tarefa no Sistema Motor e Cognitivo em Indivíduos com Doença de Parkinson. Revista Saúde e Pesquisa, 2014. 7(2): 241-249.

24. Nobrega ACL, Freitas EV, Oliveira $M A B$ et al. Posicionamento oficial da Sociedade Brasileira de Medicina do Esporte e da
Sociedade Brasileira de Geriatria e Gerontologia: Atividade Física e saúde no idoso. Revista Brasileira de Medicina no Esporte. 2008; 5(6): 207-211. 